\title{
Effects of Exenatide on Urinary Albumin in Overweight/Obese Patients with T2DM: A Randomized Clinical Trial
}

\author{
Chao Kang \\ The General Hospital of Western Theater Command \\ Qiao Qiao \\ Xinqiao Hospital \\ Qiang Tong \\ Xinqiao Hospital \\ Qian Bai \\ Xinqiao Hospital \\ Chen Huang \\ Xinqiao Hospital \\ Rong Fan \\ Xinqiao Hospital \\ Hui Wang \\ Xinqiao Hospital \\ Kanakaraju Kaliannan \\ Massachusetts General Hospital \\ Jian Wang ( $\square$ wangjianxq@hotmail.com ) \\ Xinqiao Hospital \\ Jing $X u$ \\ Xinqiao Hospital
}

\section{Research Article}

Keywords: Type 2 diabetes mellitus, Exenatide, Glycemic Control, urinary albumin

Posted Date: July 8th, 2021

DOI: https://doi.org/10.21203/rs.3.rs-616816/v1

License: (c) This work is licensed under a Creative Commons Attribution 4.0 International License. Read Full License 


\section{Abstract}

In this study, we investigated the effect of exenatide (EXE), a glucagon-like peptide (GLP)-1 receptor agonist, on kidney function, obesity indices, and glucose control in overweight/obese patients with type 2 diabetes mellitus (T2DM). A total of 159 overweight/obese patients with T2DM were randomized to the EXE group or insulin glargine (GLAR) control group for a total treatment period of 24 weeks. EXE intervention significantly reduced the UAC at both week 12 and week 24 endpoints. The levels of the anthropometric parameters and glucose parameters, lipid parameters (TG and HDL-c), and inflammation biomarkers (CRP and TNF-a) in the EXE group were improved at 12 weeks or 24 weeks respectively $(P<0.05)$. Meanwhile, a comparison between two groups showed significant $(P<0.05)$ changes in anthropometric parameters, glucose parameters (FBG HBA1C, C-peptide, and HOMA-IR), lipid parameters (TG and HDL-C) and serum levels of CRP, IL-6 and TNF-a. Correlation network analysis showed that FGF 21 had a more central role in improving metabolism in the EXE group, and the change of FGF 21 was significantly negatively correlated with UAC at week 12 and week 24 respectively $(r=-0.297, P=0.010 ; r=-0.294, P=0.012)$. Our results showed that EXE could help patients improve UAC, glycemic levels, and inflammatory biomarkers after a follow-up period of 24 weeks intervention, which may be partly mediating by FGF 21, indicating that EXE is an effective and safe way for albuminuria control in overweight/obese patients with T2DM.

\section{Introduction}

Obesity, especially abdominal obesity, is closely associated with Diabetic Nephropathy (DN), which is a major cause of morbidity and a key determinant of mortality in patients with type 2 diabetes mellitus (T2DM) ${ }^{1}$. A previous study reported that about $25 \%$ of diabetic patients are affected by DN, and DN is the primary cause of End-stage Renal Disease (ESRD) in developed countries ${ }^{2}$. Early DN shows glomerular nodular lesions and hyaline arteriolar renal pathological changes, and urinary albumin excretion continues to increase, resulting in microalbuminuria. However, there is no Glomerular Filtration Rate (GFR) decline but has entered the early DN stage. Therefore, microalbuminuria is a more reliable and sensitive early detection indicator for $\mathrm{DN}^{3}$.

Exenatide (EXE), a natural glucagon-like peptide 1 (GLP-1) analogs, is a synthetic incretin-mimetic peptide. Numerous studies have demonstrated that EXE improves glycemic control and reduces body weight in patients with T2DM ${ }^{4}$. The DURATION-3 trial with 456 patients demonstrated that once-weekly EXE treatment for three years significantly reduces $\mathrm{HbA1C}$ concentrations compared with insulin glargine ${ }^{5}$. Some evidence suggests that EXE may affect renal outcome beyond glucose-lowering. Four-weeks of EXE intervention could increase nitric oxide synthase (i-NOS) expression in the glomerular area and urine protein levels in streptozocintreated rats ${ }^{6}$. Kodera showed that exendin-4 at $10 \mu \mathrm{g} / \mathrm{kg}$ body weight daily for 8 weeks could ameliorate albuminuria, glomerular hyperfiltration, glomerular hypertrophy, and mesangial matrix expansion in the diabetic rats, as well as decreasing oxidative stress and nuclear factor-KB activation in kidney tissue ${ }^{7}$. A large Scandinavian Cohort Study showed that 38,731 new users of GLP-1 receptor agonists (liraglutide $92.5 \%$, EXE $6.2 \%$, lixisenatide $0.7 \%$, and dulaglutide $0.6 \%$ ) had a lower risk of serious renal events ${ }^{8}$. However, Tonneijck demonstrated that acute intravenous administration of EXE did not affect gold-standard-measured GFR (Glomerular Filtration Rate) and ERPF (Effective Renal Plasma Flow) in T2DM 9.

Moreover, a retrospective analysis of an observational study showed that EXE twice daily did not change kidney function or albuminuria when compared with insulin glargine. However, this observational study has some limitations in that the method of data capture did not provide information on dosage or medication adherence ${ }^{10}$. Therefore, more robust evidence needs to be confirmed. To date, no randomized study evaluates the effect of EXE on microalbuminuria in humans, and the renoprotective effect of EXE in overweight/obese patients with T2DM remains unclear.

In summary, we designed a randomized clinical trial experiment lasting for 24 weeks to assess the renoprotective effect of EXE in overweight/obese patients with T2DM. We also evaluated the impact on glycemic control, lipid metabolism, obesity indices, systematic inflammation biomarkers, and safety. Besides, we unveiled a novel role of FGF21 (Fibroblast Growth Factor 21) in the renoprotective effect of EXE.

\section{Subjects, Materials And Methods Study design}


The clinical trial was approved by the Ethical Committee of Xinqiao Hospital of Army Medical University (16-03502) and registered at http://www.chictr.org/cn/ (ChiCTR-IPR-17010825, date of registration: 09/03/2017, principal investigator: Jing Xu). The study was conducted from March to December 2017. We initially recruited the subjects at the department of endocrinology of Xinqiao Hospital, Chongqing, China. Written informed consent was obtained from all subjects participating in the trial. The study was conducted following the principles of the Declaration of Helsinki (More details of the ethics statement are shown in Supplementary Materials).

All the subjects were required to fulfill the following criteria to be eligible for our study: (1) Glycated hemoglobin (HbA1c) $\geq 6.5 \%$; (2) Body mass index (BMI) $\geq 24 \mathrm{~kg} / \mathrm{m}^{211}$; (3) Age 18-65 y; (4) Systolic blood pressure (SBP) from 90 to $120 \mathrm{~mm} \mathrm{Hg}$, and diastolic blood pressure (DBP) between 60 and $90 \mathrm{~mm} \mathrm{Hg}$; (5) Agents such as ACE inhibitors and angiotensin receptor blockers were not allowed during the study. Exclusion criteria were as follows: cancer; cardiovascular; gastrointestinal, respiratory; kidney or liver disease diagnosis or treatment; eating disorders, psychological disorders or cognitive deficit resulting in an inability to understand or comply with instructions; lactating, pregnant, or planning pregnancy before the end of the intervention; any severe illness not otherwise specified that would interfere with the participant; smoking; alcoholism; or attending another clinical trial; lack of informed consent; and judgment of the investigator that an individual is ineligible for inclusion in the study.

Eligible patients were randomized in equal proportions to EXE and insulin glargine group (GLAR). EXE was initiated at a dose of $5 \mu \mathrm{g}$ b.i.d., subcutaneously injected 15 min before breakfast and dinner. Then, EXE was up-titrated to $10 \mu \mathrm{g}$ b.i.d. for the remaining weeks of the randomized, placebo-controlled phase ${ }^{12}$. Insulin glargine was subcutaneously injected once a day. Randomization was done centrally by computer-generated randomization lists. The participants, investigators, and the sponsor's clinical team were blinded to treatment allocation. The study protocol was deposited in protocols.io (DOI: dx.doi.org/10.17504/protocols.io.bgsyjwfw) and More details of the protocol are shown in Supplementary Materials.

\section{Main Outcomes and Measures}

All subjects had fasted for 8 hours on the day of clinical examination. The primary outcome variable was the urine albumin concentration (UAC) measured using a Beckman Coulter UniCelDxC800 Synchron Clinical System. The microalbuminuria was defined as a UAC of 20 to $300 \mathrm{mg} / \mathrm{L}$ by morning urine collection, as previously reported ${ }^{13}$. Secondary outcomes included: (1) Height, weight, BMI, waist-hip ratio (WHR), and visceral fat area (VFA) measurements were taken in a laboratory between 8:00-11:00 am, after a minimum of 12 hours of fasting by using the In-Body (720) body composition analyzer (Biospace Co., Ltd., Seoul, Korea); (2) Fasting blood glucose (FBG) and $\mathrm{HbA1C}$ were measured by using a blood glucose meter (Bayer, Germany) and automatic biochemical analyzer (Hitachi 7600-020, Japan). Serum insulin was determined by an electrochemiluminescence immunoassay with an automated immunoassay analyzer (E170; Roche). C-peptide was measured by immunoradiometric assay (Centaur; Bayer Diagnostics, Mijdrecht, Netherlands). HOMA-IR values were calculated with the use of the following formula: HOMA-IR $=$ [glucose $(\mathrm{mmol} / \mathrm{L}) \times$ insulin $(\mu \mathrm{lU} / \mathrm{mL})] / 22.5$; (3) Triglycerides (TG), total cholesterol (TCH), low-density lipoprotein cholesterol (LDL-c), high-density lipoprotein cholesterol (HDL-C) were detected by using automatic biochemical analyzer (Hitachi 7600-020, Japan); (4) C-reactive protein (CRP), interleukin-6 (IL-6), interleukin-8 (IL-8) and tumor necrosis factor-a (TNF-a) were measured by using a multiplex xMAP technology with a Luminex 100 Analyzer (Luminex Corp., Austin, TX, USA). Serum FGF21 level was measured using a Human FGF21 Quantikine ELISA Kit (R\&D Systems). All of the samples were collected after $\geq 12 \mathrm{~h}$ of fasting. The tests are double-blind, and the quality control is carried out regularly by the inspection department.

\section{Safety and tolerability assessments}

Participants had low blood sugar and other adverse reactions such as nausea, vomiting, and dizziness. Patients with palpitations, sweating, and other discomforts were immediately assessed for the blood glucose levels. Hypoglycemia was defined as less than 3.9 $\mathrm{mmol} / \mathrm{L}$; Severe hypoglycemia was defined as either blood glucose levels of less than $3.1 \mathrm{mmol} / \mathrm{L}$ were observed, or hypoglycemic symptoms were relieved after oral administration of carbohydrates or intravenous glucose or glucagon.

\section{Statistical analyses}

This study was a randomized controlled trial with two parallel groups. A sample size of 71 patients per group was required to provide $90 \%$ power to detect a between-group significant difference in UAC between the two treatment groups, assuming that the data is analyzed on a log-scale, with 0.05 alpha level and an intrasubject coefficient of variation of 50\%. Moreover, we divide the alpha level by the number of tests being carried out for alpha adjustments of further analysis using 'longpower' R packages. R software (version 3.4.4) was used for statistical analysis unless otherwise stated. An intent-to-treat (ITT) criterion was performed with all participants, including those who withdrew before outcome determination. Between groups and within-group comparisons of continuous variables 
were conducted using linear mixed-effects models in "ImerTest" and "emmeans" packages in R. Time was treated as a categorical variable. The models included group and time as fixed covariates, as well as the group $\times$ time interactions, with gender as covariates.

Random intercepts for subjects accounted for the dependence of repeated measures ${ }^{14}$. To evaluate the size of the difference between and within the groups, we performed the effect size (ES) that reflects changes in SD units. Correlation-based network analysis was performed among metabolic parameters adjusted for age and gender were calculated with Pearson's correlations coefficient on residuals from regression models, including the adjustment variables for each pair of biomarkers. The Pearson correlation was used to estimate correlation coefficients. Threshold tests for correlation coefficients ( $r$ ) and q-values were applied to detect significant correlations. Thresholds were set for $\mathrm{q}$ of $\leq 0.05$. Spurious correlations were removed, while significant correlations were transformed into network form. All statistical tests were 2-tailed and, unless otherwise noted, $P<0.05$ was considered the level of statistical significance. Variables are expressed as frequencies with percentages, median (IQRs), and means (SD).

\section{Results}

\section{Participant Characteristics}

A total of 1 two groups were enrolled in 79 and 80 , respectively. At the end of the intervention, seven patients in the EXE group and four patients in the GLAR group withdrew. The reason for the loss of follow-up was that the patients did not return to the outpatient department for blood sampling because of an unexpected lack of interest during the study (Figure 1). There were no significant differences between the two groups in age, gender, duration of diabetes, prestudy antidiabetic treatment, and albuminuria before intervention (Table 1). After EXE treatment, 19 patients (26.39\%) had mild or higher gastrointestinal adverse reactions. One patient with moderate adverse reactions was relieved spontaneously with the treatment cycle's extension, without other adverse severe reactions.

\section{Primary End Point}

We conducted analyses to identify differences within and between groups of UAC during the study periods. EXE intervention could significantly reduce the UAC at both week 12 and week 24 endpoint, while Insulin glargine did not significantly influence the UAC $(P<0.05$, Figure 2A). Linear mixed-effects models analysis revealed that subjects in the EXE group had significantly improved UAC than those in the GLAR group at week $24(P<0.05$, Figure $2 \mathrm{~B})$.

\section{Other Efficacy Variables}

The anthropometric parameters (weight, BMI, WHR, VFA) and glucose parameters and biomarkers of lipid profile and inflammation biomarkers (CRP, TNF- $a$ and II-6) showed no significant differences between the two groups at baseline. The anthropometric parameters (weight, BMI, WHR, VFA), glucose parameters (FBG, HBA1C, insulin and HOMA-IR), lipid parameters (HDL-C), and inflammation biomarkers such as TNF-a were significantly improved at both week 12 or week 24 endpoints in EXE group $(P<0.05$, Table 2). Meanwhile, the comparison between groups showed that changes of anthropometric parameters (weight, BMI, WHR, VFA), glucose parameters (FBG HBA1C, C-peptide and HOMA-IR), lipid parameters (TG and HDL-C), and biomarkers of inflammation profile (CRP, IL-6 and TNF-a) of patients in the EXE group were significantly different from those in another group at week 12 or week $24(P<0.05$, Table 2$)$.

\section{Correlations between FGF 21 and other efficacy variables}

To explore the mechanism of the renoprotective effect of EXE, we measured the level of serum FGF21. EXE significantly increased FGF 21 at week $24(P<0.05$, Figure $2 C)$. Between-group comparison uncovered that EXE group subjects had significantly improved FGF 21 level than those in the GLAR group at week 24 by using linear mixed-effects models analysis $(P<0.05$, Figure 2D).

We then explored correlation-based network analysis among UAC, anthropometric parameters, glucose and lipid metabolism parameters, inflammation parameters, and FGF 21. The overall results demonstrated more associations between FGF 21 and other metabolic parameters in the EXE group when compared with the GLAR group (Figure 3). Detailed correlation analysis showed that the estimated change of FGF 21 was negatively correlated with UAC (Week 12: $r=-0.297, P=0.010$; Week 24: $r=-0.294, P=0.012$ ), Weight (Week 12: $r=-0.336, P=0.003$; Week 24: $r=-0.337, P=0.004$ ), VFA (Week 12: $r=-0.273, P=0.018$; Week 24: $r=-0.281, P=0.016$ ) and HBA1C (Week 12: $r=-0.340, P=0.003$; Week 24: $r=-0.365, P=0.002$ ) in EXE group at both week 12 and week 24 (Supplementary Figure 1A-B). However, a few correlations between FGF 21 and WHR or TCH was found in the GLAR group. These results indicated that FGF 21 might play an important role in improving EXE on urine albumin and other metabolic parameters. 


\section{Discussion}

We completed a 24-week, prospective, randomized controlled trial of the EXE intervention in overweight/obese patients with T2DM. The main finding of our study was that 24-week EXE intervention significantly reduced UAC, which is a biomarker for DN. The results also showed that the patients in the EXE group present more significant improvements in weight and glucose parameters (FBG, $\mathrm{HbA1C}$ ). These data indicate that using EXE among overweight/obese patients with T2DM is more helpful than traditional outpatient services for better UAC and glycemic control.

Abdominal obesity can lead to glucose metabolism disorders and atherosclerosis, which has a more significant impact on the metabolic level and leads to insulin resistance ${ }^{15}$. Our results showed that a decrease in UAC is positively correlated with the change of WHR, indicating that abdominal obesity is more closely related to DN compared with systemic obesity. Furthermore, as reported previously, the correlation between abdominal obesity and DN is independent of known risk factors such as BMI and age, diabetes course, and blood pressure $1,16,17$. Weight loss could also reduce urinary albumin excretion and prevent glomerular filtration in obese patients with $\mathrm{DN}{ }^{18}$. Besides, large prospective studies on type 2 diabetes have respectively verified that strict control of blood glucose could reduce the occurrence or delay the progression of DN in patients with diabetes, indicating that glycemic control is also an essential factor for Microalbuminuria in T2DM ${ }^{19}$. Therefore, losing weight, especially improving abdominal obesity and lowering blood glucose, has great significance in delaying DN development. We also found that the serum levels of TG and HDL-c were improved accordantly with the previous result ${ }^{20}$. However, Tch and LDL-c levels were not changed, which may be due to either the small sample size or the duration of intervention, or both. This study used WHR and VFA to assess abdominal obesity and found that EXE dramatically improved abdominal obesity, which has a positive relationship with UAC.

Microalbuminuria is the earliest biochemical parameter of DN and the important risk factor affecting DN progression and cardiovascular diseases. In line with previous studies, our results showed that EXE dramatically improved UAC, a well-known biomarker of microalbuminuria ${ }^{21}$. EXE is a synthetic version of exendin-4 and displays biological properties similar to human GLP-1, produced by intestinal $L$ endocrine cells when ingestion of food ${ }^{4}$. A previous study showed that Exendin-4 ameliorated albuminuria, glomerular hyperfiltration in diabetic rats via decreasing oxidative stress and nuclear factor-kB activation in kidney tissue. Also, it reduced proinflammatory cytokines from macrophages on glomerular endothelial cells ${ }^{7}$. After 16 weeks of treatment in T2DM patients, EXE attenuated urinary excretion of TGF-1 and type IV collagen, which has been proven to correlate with microalbuminuria severity ${ }^{21}$. The mRNA expression of TNF- $a$, IL-1 $\beta$, JNK-1, TLR-2, TLR-4, and SOCS-3 in mononuclear cells and monocyte plasma concentrations chemoattractant protein-1, matrix metalloproteinase-9, serum amyloid A, and IL-6 of 12 patients was significantly decreased after 12 weeks of EXE treatment ${ }^{22}$. Dandona reported that EXE increased the Interleukin-1 receptor antagonist (IL-1RA) via the Nrf2-Keap1-ARE system to suppress inflammatory and oxidative stress ${ }^{23}$. Moreover, activation of Nrf2-Keap1 could suppress oxidative stress to protect renal function ${ }^{24}$. These results indicated that EXE could improve urinary microalbuminuria by reducing inflammation and oxidative stress, suggesting a pioneering role of EXE in renal protection and against the progression of DN. However, few clinical studies evaluated the impact of EXE on inflammation. Here, we identified that EXE could reduce inflammation parameters while improving microalbumin in overweight/obese patients with T2DM. Numerous studies have uncovered that FGF21 causes considerable pharmacological benefits on metabolic diseases, such as hyperglycemia, insulin resistance, dyslipidemia, and other obesity-related diseases ${ }^{25}$. Here, our results showed that EXE could significantly increase the serum level of FGF 21, which is negatively correlated with the change of UAC. Liu showed that FGF 21 is upregulated by EXE in both hepatocytes and humans, and the increase of FGF21 was blocked in Fgf21 knockout mice ${ }^{26}$. Nevertheless, no study has assessed the correlation between FGF 21 and the renoprotective effect of EXE in humans.

A previous meta-analysis study concluded that both UAC and UACR yielded high sensitivity and specificity to detect microalbuminuria in patients with diabetes mellitus after collecting and carefully analyzing 14 published studies ${ }^{27}$. Here, we used UAC as the biomarker for microalbuminuria due to its convenient use and low cost for patients. Moreover, due to the limitation of the study period, whether the improvement in urinary microalbumin could be maintained needs further evaluation. Likewise, whether the VFA and weight of patients would rise for a short time after the withdrawal of EXE or the necessity of changes in hypoglycemic regimentation needs to be further evaluated.

In conclusion, our results showed that EXE could help patients improve UAC and glycemic levels and inflammatory biomarkers after a follow-up period of 24 weeks intervention, indicating that EXE is the effective and safe way for albuminuria control in diabetic patients. 
We first showed that the increase of FGF 21 is correlated with the decrease of UAC after EXE intervention, providing that FGF 21 may be involved in the renoprotective effect of EXE. Our results could help the clinician to pay more attention to the renoprotective effects of EXE when treating diabetes, especially those with obesity. These findings may provide new options for patients with T2DM seeking better albuminuria and glycemic control.

\section{Declarations}

\section{Disclosure Statement:}

The authors have declared no conflict of interest.

This research was supported by Project 2014YLC20 of the Xinqiao Hospital, Project ctstc2015shmszx120014, and ctstc2015jcsf10003 of the Chongqing Science and Technology Commission.

\section{Acknowledgments}

This research was supported by Project 2014YLC20 of the Xinqiao Hospital, Project ctstc2015shmszx120014, and ctstc2015jcsf10003 of the Chongqing Science and Technology Commission. The authors' responsibilities were as follows: JX and JW designed the experiments; CK, QQ, QT, QB, CH, RF, and HW collected samples and performed the experiments; $\mathrm{CK}, \mathrm{QQ}, \mathrm{KK}$, and JX analyzed the data; CK, QQ, KK, JX, and JW prepared the manuscript and had primary responsibility for final content; and all authors read and approved the final manuscript. None of the authors reported a conflict of interest.

\section{References}

1. Hu, J.et al. Abdominal Obesity Is More Closely Associated With Diabetic Kidney Disease Than General Obesity. Diabetes care 39, e179-180, doi:10.2337/dc16-1025 (2016).

2. de Boer, I. H.et al. Temporal trends in the prevalence of diabetic kidney disease in the United States. JAMA 305, 2532-2539 (2011).

3. KDOQI. KDOQI Clinical Practice Guidelines and Clinical Practice Recommendations for Diabetes and Chronic Kidney Disease. American journal of kidney diseases : the official journal of the National Kidney Foundation 49, S12-154 (2007).

4. Meier, J. J. GLP-1 receptor agonists for individualized treatment of type 2 diabetes mellitus. Nat Rev Endocrino/ 8, 728-742 (2012).

5. Diamant, M.et al. Exenatide once weekly versus insulin glargine for type 2 diabetes (DURATION-3): 3-year results of an open-label randomised trial. The lancet. Diabetes \& endocrinology 2, 464-473 (2014).

6. Çavusoglu, T.et al. Comparison of nephron-protective effects of enalapril and GLP analogues (exenatide) in diabetic nephropathy. Experimental and clinical endocrinology \& diabetes : official journal, German Society of Endocrinology [and] German Diabetes Association 122, 327-333 (2014).

7. Kodera, R.et al. Glucagon-like peptide-1 receptor agonist ameliorates renal injury through its anti-inflammatory action without lowering blood glucose level in a rat model of type 1 diabetes. Diabetologia 54, 965-978 (2011).

8. Pasternak, B.et al. Use of Glucagon-Like Peptide 1 Receptor Agonists and Risk of Serious Renal Events: Scandinavian Cohort Study. Diabetes care 43, 1326-1335 (2020).

9. Tonneijck, L.et al. Acute renal effects of the GLP-1 receptor agonist exenatide in overweight type 2 diabetes patients: a randomised, double-blind, placebo-controlled trial. Diabetologia 59, 1412-1421 (2016).

10. Pawaskar, M., Tuttle, K. R., Li, Q., Best, J. H. \& Anderson, P. W. Observational study of kidney function and albuminuria in patients with type 2 diabetes treated with exenatide BID versus insulin glargine. The Annals of pharmacotherapy 48, 571-576 (2014).

11. Association, A. D. Classification and Diagnosis of Diabetes: Standards of Medical Care in Diabetes-2019. Diabetes care 42, S13s28 (2019).

12. Kelly, A. S.et al. The effect of glucagon-like peptide-1 receptor agonist therapy on body mass index in adolescents with severe obesity: a randomized, placebo-controlled, clinical trial. JAMA pediatrics 167, 355-360 (2013).

13. Bouhanick, B.et al. Predictive value of testing random urine sample to detect microalbuminuria in diabetic subjects during outpatient visit. Diabete \& metabolisme 18, 54-58 (1992).

Page 6/14 
14. Detry, M. A. \& Ma, Y. Analyzing Repeated Measurements Using Mixed Models. Jama 315, 407-408(2016).

15. Hou, X.et al. Impact of waist circumference and body mass index on risk of cardiometabolic disorder and cardiovascular disease in Chinese adults: a national diabetes and metabolic disorders survey. PLoS One 8, e57319, doi:10.1371/journal.pone.0057319 (2013).

16. Viswanathan, P.et al. Exenatide therapy in obese patients with type 2 diabetes mellitus treated with insulin. Endocrine practice : official journal of the American College of Endocrinology and the American Association of Clinical Endocrinologists 13, 444-450 (2007).

17. Chaudhuri, A.et al. Exenatide induces an increase in vasodilatory and a decrease in vasoconstrictive mediators. Diabetes, obesity \& metabolism 19, 729-733 (2017).

18. Saiki, A.et al. Effect of weight loss using formula diet on renal function in obese patients with diabetic nephropathy. International journal of obesity 29, 1115-1120 (2005).

19. Adler, A. I.et al. Development and progression of nephropathy in type 2 diabetes: the United Kingdom Prospective Diabetes Study (UKPDS 64). Kidney international 63, 225-232 (2003).

20. Patti, A. M.et al. Exenatide once-weekly improves metabolic parameters, endothelial dysfunction and carotid intima-media thickness in patients with type-2 diabetes: An 8-month prospective study. Diabetes research and clinical practice 149, 163-169 (2019).

21. Zhang, H., Zhang, X., Hu, C. \& Lu, W. Exenatide reduces urinary transforming growth factor-beta1 and type IV collagen excretion in patients with type 2 diabetes and microalbuminuria. Kidney \& blood pressure research 35, 483-488 (2012).

22. Chaudhuri, A. et al. Exenatide exerts a potent antiinflammatory effect. The Journal of clinical endocrinology and metabolism 97 , 198-207 (2012).

23. Dandona, P.et al. Exenatide Increases IL-1RA Concentration and Induces Nrf-2-Keap-1-Regulated Antioxidant Enzymes: Relevance to $\beta$-Cell Function. The Journal of clinical endocrinology and metabolism 103, 1180-1187 (2018).

24. Nezu, M., Suzuki, N. \& Yamamoto, M. Targeting the KEAP1-NRF2 System to Prevent Kidney Disease Progression. American journal of nephrology 45, 473-483 (2017).

25. Geng, L., Lam, K. S. L. \& Xu, A. The therapeutic potential of FGF21 in metabolic diseases: from bench to clinic. Nature reviews. Endocrinology 16,654-667 (2020).

26. Liu, J.et al. Liver-derived fibroblast growth factor 21 mediates effects of glucagon-like peptide-1 in attenuating hepatic glucose output. EBioMedicine 41, 73-84 (2019).

27. Wu, H. Y.et al. Diagnostic performance of random urine samples using albumin concentration vs ratio of albumin to creatinine for microalbuminuria screening in patients with diabetes mellitus: a systematic review and meta-analysis. JAMA internal medicine $174,1108-1115$ (2014).

\section{Tables}

Table 1. Baseline characteristics of the two groups. 


\begin{tabular}{|lll|}
\hline Variable name & EXE $(\mathbf{n}=79)$ & GLAR $(\mathbf{n}=80)$ \\
\hline Age (years) & $49.37 \pm 8.43$ & $47.63 \pm 9.65$ \\
\hline Gender: men, No. (\%) & $42(53.16)$ & $45(56.25)$ \\
\hline Duration of diabetes (years) & $3.0(1.0,5.0)$ & $3.0(2.0,4.0)$ \\
\hline Prestudy antidiabetic treatment, No. (\%) & & \\
\hline Metformin and sulphonylurea combination & $42(53.16)$ & $48(60.00)$ \\
\hline Sulphonylurea alone & $10(12.66)$ & $12(15.00)$ \\
\hline Metformin alone & $27(34.18)$ & $20(25.00)$ \\
\hline UAC & & \\
\hline Median (IQRs), mg/L & $80.0(50.0,150.0)$ & $80.0(40.0,108.8)$ \\
\hline Geometric mean (CV\%), mg/L & $80.60(84.86 \%)$ & $69.59(86.94 \%)$ \\
\hline Albuminuria, No. (\%) & & 7 \\
\hline Normal (<20 mg/L) & 5 & 72 \\
\hline High (>20-<300 mg/L) & 73 & 1 \\
\hline Very high ( $\geq 300 \mathrm{mg} / \mathrm{L})$ & 1 & \\
\hline
\end{tabular}

Data are presented as frequencies with percentages, median (IQRs), and mean \pm SD. Abbreviations: UAC, urine albumin concentration.

Table 2. Baseline value and estimate change in the anthropometric composition parameters, biochemical variables and inflammation biomarkers in two groups at 12 and 24 weeks. 
ITT Analysis of EXE Within Group
ITT Analysis of GLAR

Within Group

\section{Between Group}

Mean(SD)

Value
ES Mean(SD)

\begin{abstract}
Value
\end{abstract}
ES

$P-$
Value $\quad 95 \% \mathrm{Cl}$

ES

\section{Anthropometric parameters}

Weight

(Kg)

\begin{tabular}{|c|c|c|c|c|c|c|c|c|c|}
\hline Baseline & $75.241(8.167)$ & & & $73.575(6.106)$ & & & 0.123 & -0.312 to 2.585 & 1.136 \\
\hline $\begin{array}{l}\Delta \text { Week } \\
12\end{array}$ & $-2.969(8.148)$ & $<0.001$ & 2.187 & $-0.414(6.043)$ & 0.141 & 0.302 & 0.309 & -2.197 to 0.701 & -0.748 \\
\hline $\begin{array}{l}\Delta 4 \text { Week } \\
24\end{array}$ & $-5.388(8.334)$ & $<0.001$ & 3.720 & $-0.658(5.915)$ & 0.221 & 0.269 & 0.002 & -3.773 to -0.857 & -2.315 \\
\hline \multicolumn{10}{|l|}{$\begin{array}{l}\mathrm{BMI}, \\
\mathrm{kg} / \mathrm{m}^{2}\end{array}$} \\
\hline Baseline & $27.607(2.027)$ & & & $27.064(1.937)$ & & & 0.084 & -0.132 to 2.056 & 0.962 \\
\hline $\begin{array}{l}\Delta \text { Week } \\
12\end{array}$ & $-1.160(2.041)$ & $<0.001$ & 2.204 & $-0.120(1.897)$ & 0.130 & 0.308 & 0.094 & -2.029 to 0.163 & -0.933 \\
\hline $\begin{array}{l}\Delta \text { Week } \\
24\end{array}$ & $-2.005(2.078)$ & $<0.001$ & 3.734 & $-0.060(1.882)$ & 0.200 & 0.277 & $<0.001$ & -3.603 to -1.385 & -2.494 \\
\hline \multicolumn{10}{|l|}{ WHR } \\
\hline Baseline & $0.901(0.041)$ & & & $0.898(0.032)$ & & & 0.578 & -0.421 to 0.752 & 0.166 \\
\hline $\begin{array}{l}\Delta \text { Week } \\
12\end{array}$ & $-0.015(0.039)$ & $<0.001$ & 0.744 & $-0.007(0.033)$ & 0.046 & 0.379 & 0.508 & -0.789 to 0.392 & -0.199 \\
\hline $\begin{array}{l}\Delta \text { Week } \\
24\end{array}$ & $-0.031(0.039)$ & $<0.001$ & 1.628 & $-0.010(0.033)$ & 0.000 & 0.653 & 0.008 & -1.406 to -0.212 & -0.809 \\
\hline \multicolumn{10}{|l|}{$\operatorname{VFA}\left(\mathrm{m}^{2}\right)$} \\
\hline Baseline & $104.667(25.236)$ & & & $104.075(17.198)$ & & & 0.856 & -0.656 to 0.789 & 0.067 \\
\hline $\begin{array}{l}\Delta \text { Week } \\
12\end{array}$ & $-14.492(24.636)$ & $<0.001$ & 1.721 & $-0.976(17.211)$ & 0.849 & 0.087 & $<0.001$ & -2.301 to -0.834 & -1.568 \\
\hline $\begin{array}{l}\Delta \text { Week } \\
24\end{array}$ & $-24.912(24.482)$ & $<0.001$ & 2.869 & $-3.117(17.511)$ & 0.102 & 0.331 & $<0.001$ & -3.219 to -1.724 & -2.471 \\
\hline
\end{tabular}

\section{Parameter of glucose metabolism}

FBG

$(\mathrm{mmol} / \mathrm{L})$

\begin{tabular}{llllllllll} 
Baseline & $9.916(2.157)$ & & & $9.578(1.633)$ & & & 0.164 & -0.116 to 0.678 & 0.281 \\
$\begin{array}{llllll}\Delta \text { Week } \\
12\end{array}$ & $-2.338(2.154)$ & $<0.001$ & 1.845 & $-1.007(1.645)$ & $<0.001$ & 0.826 & 0.000 & -1.143 to -0.333 & -0.738 \\
\hline $\begin{array}{l}\Delta \text { Week } \\
24\end{array}$ & $-3.063(2.136)$ & $<0.001$ & 2.385 & $-1.780(1.658)$ & $<0.001$ & 1.419 & 0.001 & -1.094 to -0.275 & -0.685
\end{tabular}

24

HBA1C

(\%)

\begin{tabular}{|c|c|c|c|c|c|c|c|c|c|}
\hline Baseline & $9.726(1.974)$ & & & $9.644(1.545)$ & & & 0.874 & $\begin{array}{l}-0.301 \text { to } \\
0.3541\end{array}$ & 0.027 \\
\hline $\begin{array}{l}\Delta{ }_{12} \text { Week } \\
\text {. }\end{array}$ & $-1.760(2.016)$ & 0.001 & 0.580 & $0.184(1.550)$ & 0.930 & -0.058 & 0.000 & -0.947 to -0.276 & -0.612 \\
\hline
\end{tabular}




\begin{tabular}{|c|c|c|c|c|c|c|c|c|c|}
\hline $\begin{array}{l}\Delta \text { Week } \\
24\end{array}$ & $-2.574(2.020)$ & $<0.001$ & 0.823 & $-1.562(1.513)$ & 0.004 & 0.515 & 0.103 & $\begin{array}{l}-0.620 \text { to } \\
0.0573\end{array}$ & -0.282 \\
\hline \multicolumn{10}{|c|}{ C-peptide (nmol/L) } \\
\hline Baseline & $1.352(0.452)$ & & & $1.309(0.386)$ & & & 0.538 & -0.239 to 0.458 & 0.109 \\
\hline $\begin{array}{l}\Delta \text { Week } \\
12\end{array}$ & $0.652(0.427)$ & $<0.001$ & -1.556 & $0.151(0.381)$ & 0.065 & -0.357 & $<0.001$ & 0.944 to 1.672 & 1.308 \\
\hline $\begin{array}{l}\Delta \text { Week } \\
24\end{array}$ & $1.039(0.427)$ & $<0.001$ & -2.431 & $0.338(0.388)$ & $<0.001$ & -0.780 & $<0.001$ & 1.383 to 2.138 & 1.761 \\
\hline \multicolumn{10}{|c|}{ Insulin (mU/L) } \\
\hline Baseline & $9.949(3.080)$ & & & $9.491(2.920)$ & & & 0.198 & -0.115 to 0.552 & 0.218 \\
\hline$\underset{12}{\Delta \text { Week }}$ & $-1.723(3.112)$ & $<0.001$ & 0.768 & $-0.473(2.940)$ & 0.364 & 0.216 & 0.053 & -0.673 to 0.005 & -0.334 \\
\hline $\begin{array}{l}\Delta \text { Week } \\
24\end{array}$ & $-2.812(3.145)$ & $<0.001$ & 1.282 & $-1.988(2.990)$ & $<0.001$ & 0.935 & 0.461 & -0.473 to 0.215 & -0.129 \\
\hline \multicolumn{10}{|l|}{ HOMA-IR } \\
\hline Baseline & $4.320(1.620)$ & & & $4.056(1.547)$ & & & 0.141 & $\begin{array}{l}-0.083 \text { to } \\
0.5808\end{array}$ & 0.249 \\
\hline $\begin{array}{l}\Delta \text { Week } \\
12\end{array}$ & $-1.488(1.618)$ & $<0.001$ & 1.318 & $-0.621(1.561)$ & 0.001 & 0.571 & 0.004 & -0.836 to -0.159 & -0.497 \\
\hline $\begin{array}{l}\Delta \text { Week } \\
24\end{array}$ & $-2.190(1.624)$ & $<0.001$ & 1.947 & $-1.466(1.585)$ & $<0.001$ & 1.340 & 0.040 & -0.702 to -0.015 & -0.359 \\
\hline
\end{tabular}

\section{Parameter of lipid metabolism}

$\mathrm{TCH}(\mathrm{mmol} / \mathrm{L})$

\begin{tabular}{llllllllll} 
Baseline & 4.863(1.012) & & & $4.952(0.850)$ & & & 0.558 & -0.410 to 0.222 & -0.094 \\
$\begin{array}{l}\Delta \text { Week } \\
12\end{array}$ & $-0.198(0.980)$ & 0.604 & 0.155 & $-0.125(0.842)$ & 0.655 & 0.139 & 0.502 & -0.431 to 0.212 & -0.110 \\
$\begin{array}{l}\Delta \text { Week } \\
24\end{array}$ & $-0.230(0.970)$ & 0.564 & 0.166 & $0.022(0.857)$ & 0.999 & -0.007 & 0.109 & -0.594 to -0.060 & -0.267 \\
\hline
\end{tabular}

TG

(mmol/L)

\begin{tabular}{llllllllll} 
Baseline & $2.037(1.289)$ & & & $1.974(1.131)$ & & & 0.758 & -0.264 to 0.362 & 0.049 \\
\hline $\begin{array}{l}\Delta \text { Week } \\
12\end{array}$ & $0.076(1.294)$ & 0.783 & -0.108 & $-0.463(1.134)$ & 0.066 & 0.356 & 0.002 & 0.193 to 0.832 & 0.513 \\
\hline $\begin{array}{l}\Delta \text { Week } \\
24\end{array}$ & $0.471(1.300)$ & 0.020 & -0.438 & $0.048(1.144)$ & 0.893 & -0.073 & 0.012 & 0.090 to 0.739 & 0.414 \\
\hline
\end{tabular}

LDL-c (mmol/L)

\begin{tabular}{|c|c|c|c|c|c|c|c|c|c|}
\hline Baseline & $2.745(0.698)$ & & & $2.596(0.706)$ & & & 0.145 & -0.080 to 0.544 & 0.232 \\
\hline $\begin{array}{l}\Delta \text { Week } \\
12\end{array}$ & $-0.168(0.665)$ & 0.417 & 0.204 & $0.167(0.704)$ & 0.203 & -0.271 & 0.132 & -0.561 to 0.074 & -0.243 \\
\hline $\begin{array}{l}\Delta 4 \text { Week } \\
24\end{array}$ & $-0.164(0.685)$ & 0.358 & 0.222 & $0.104(0.665)$ & 0.337 & -0.226 & 0.187 & -0.539 to 0.106 & -0.217 \\
\hline \multicolumn{10}{|c|}{ HDL-c (mmol/L) } \\
\hline Baseline & $1.389(0.452)$ & & & $1.431(0.329)$ & & & 0.552 & -0.452 to 0.242 & -0.105 \\
\hline $\begin{array}{l}\Delta \text { Week } \\
12\end{array}$ & $-0.097(0.461)$ & 0.287 & 0.244 & $0.099(0.329)$ & 0.233 & -0.259 & 0.001 & -0.964 to -0.254 & -0.609 \\
\hline
\end{tabular}




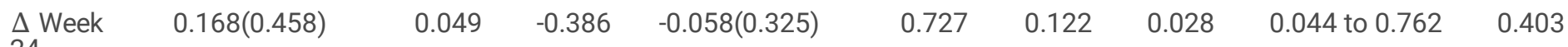

Inflammation biomarkers

CRP

$(\mathrm{mg} / \mathrm{L})$

\begin{tabular}{|c|c|c|c|c|c|c|c|c|c|}
\hline Baseline & $4.927(2.897)$ & & & $5.291(2.229)$ & & & 0.331 & -0.643 to 0.218 & -0.213 \\
\hline $\begin{array}{l}\Delta \text { Week } \\
12\end{array}$ & $-0.321(2.818)$ & 0.394 & 0.212 & $0.435(2.192)$ & 0.205 & -0.271 & 0.002 & -1.132 to -0.257 & -0.695 \\
\hline $\begin{array}{l}\Delta \text { Week } \\
24\end{array}$ & $-2.406(2.816)$ & $<0.001$ & 1.452 & $0.401(2.221)$ & 0.291 & -0.242 & $<0.001$ & -2.364 to -1.448 & -1.906 \\
\hline
\end{tabular}

IL-6

$(\mathrm{pg} / \mathrm{mL})$

\begin{tabular}{|c|c|c|c|c|c|c|c|c|c|}
\hline Baseline & $4.311(2.494)$ & & & $4.489(1.817)$ & & & 0.591 & -0.447 to 0.255 & -0.096 \\
\hline $\begin{array}{l}\Delta \text { Week } \\
12\end{array}$ & $0.254(2.523)$ & 0.515 & -0.178 & $0.211(1.828)$ & 0.666 & -0.137 & 0.763 & -0.411 to 0.302 & -0.055 \\
\hline $\begin{array}{l}\Delta \text { Week } \\
24\end{array}$ & $-0.311(2.545)$ & 0.691 & 0.134 & $0.296(1.841)$ & 0.430 & -0.199 & 0.020 & -0.792 to -0.066 & -0.429 \\
\hline
\end{tabular}

IL-

$8(\mathrm{pg} / \mathrm{mL})$

\begin{tabular}{|c|c|c|c|c|c|c|c|c|c|}
\hline Baseline & $7.265(1.473)$ & & & $7.059(1.187)$ & & & 0.413 & -0.218 to 0.530 & 0.156 \\
\hline $\begin{array}{l}\Delta \text { Week } \\
12\end{array}$ & $0.183(1.489)$ & 0.727 & -0.123 & $-0.053(1.191)$ & 0.962 & 0.042 & 0.097 & -0.059 to 0.701 & 0.321 \\
\hline $\begin{array}{l}\Delta \text { Week } \\
24\end{array}$ & $0.292(1.500)$ & 0.446 & -0.198 & $0.278(1.145)$ & 0.2266 & -0.250 & 0.594 & -0.281 to 0.489 & 0.104 \\
\hline
\end{tabular}

TNF-a $(\mathrm{pg} / \mathrm{mL})$

\begin{tabular}{|c|c|c|c|c|c|c|c|c|c|}
\hline Baseline & 4.856(1.719) & & & $4.748(1.572)$ & & & 0.711 & -0.324 to 0.475 & 0.075 \\
\hline $\begin{array}{l}\Delta \text { Week } \\
12\end{array}$ & $1.616(1.715)$ & $<0.001$ & -1.196 & $0.059(1.565)$ & 0.925 & -0.060 & $<0.001$ & 0.800 to 1.624 & 1.212 \\
\hline $\begin{array}{l}\Delta \text { Week } \\
24\end{array}$ & $2.882(1.599)$ & $<0.001$ & -2.017 & $0.164(1.586)$ & 0.721 & -0.124 & $<0.001$ & 1.539 to 2.398 & 1.969 \\
\hline
\end{tabular}

Data are presented as mean (SD). Between-group and Within-group comparisons were analyzed by Linear mixed-effects model analysis. Abbreviations: BMI, body mass index; WHR, waist-hip ratio; VFA, visceral fat area; FBG, fasting blood glucose; HbA1C, glycosylated hemoglobin; TCH, total cholesterol; TG, triglycerides; LDL-c, low-density lipoprotein cholesterol, HDL-c, high-density lipoprotein cholesterol; CRP, C-reactive protein; IL-6, interleukin-6; IL-8, interleukin-8; TNF-a, tumor necrosis factor-a.

Figures 


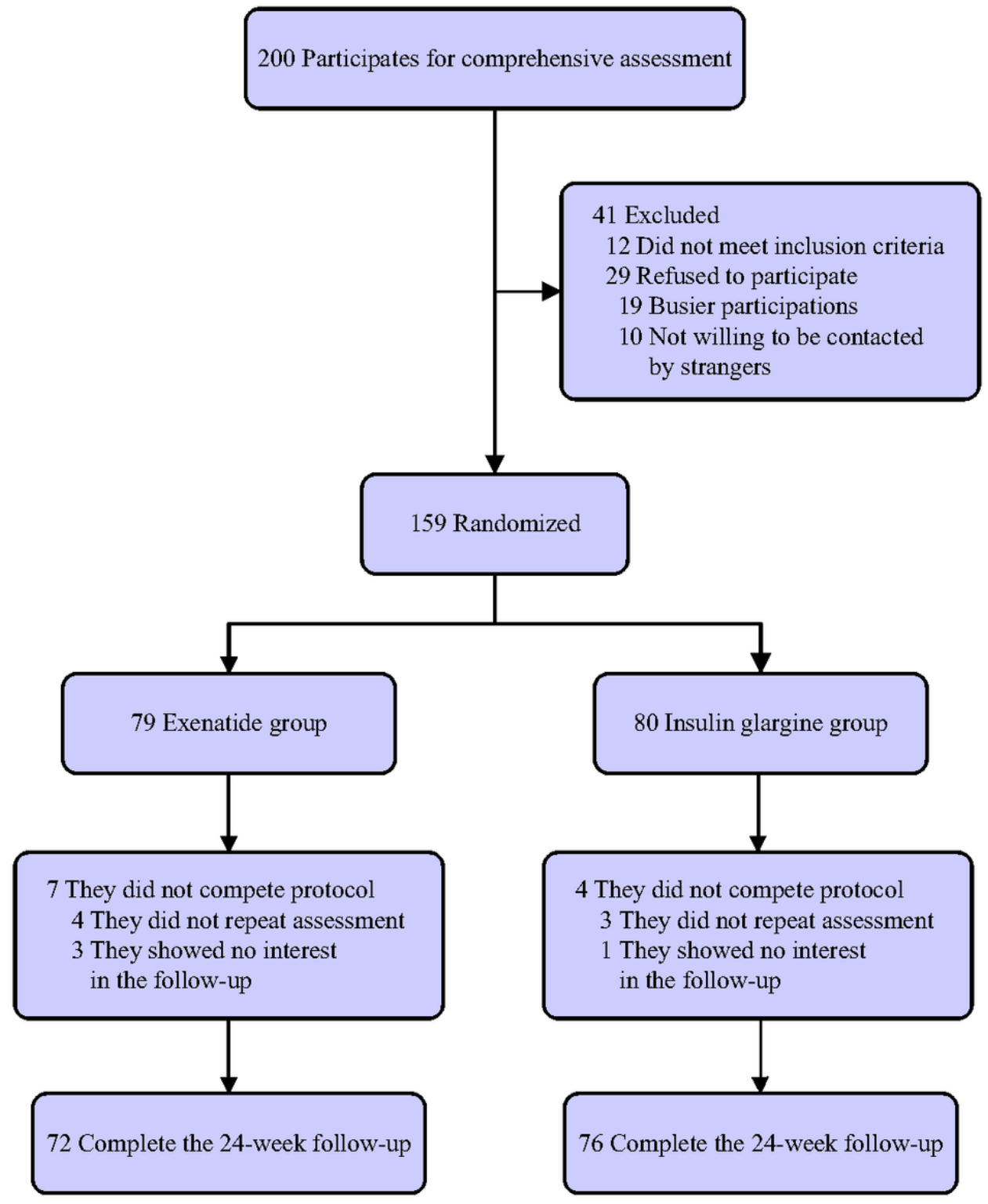

\section{Figure 1}

Flow diagram of the study participants. 
A

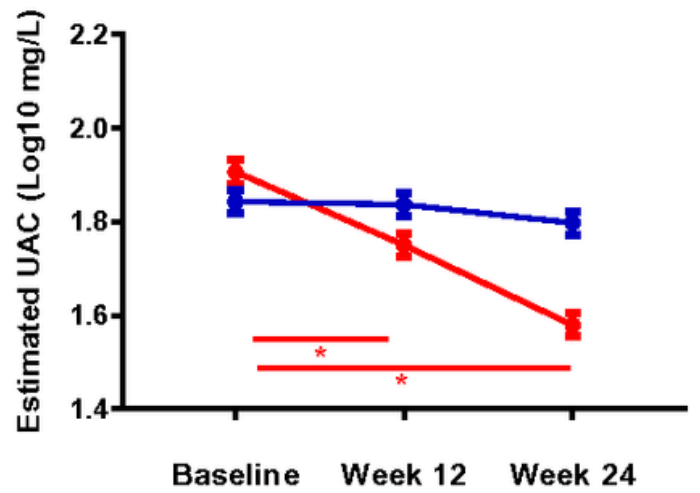

C

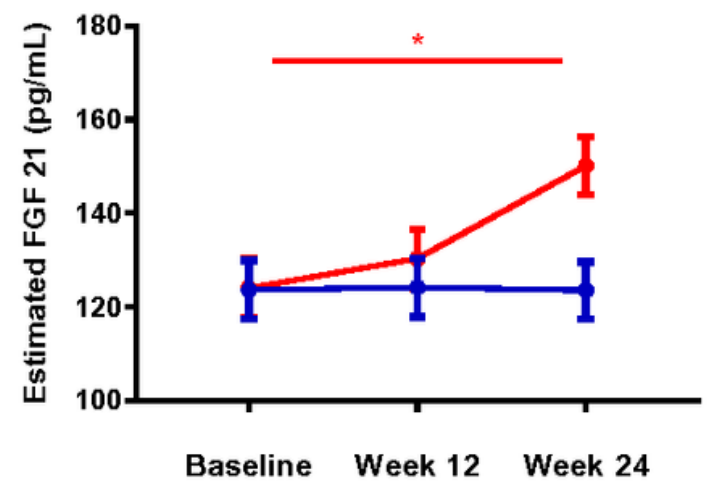

B

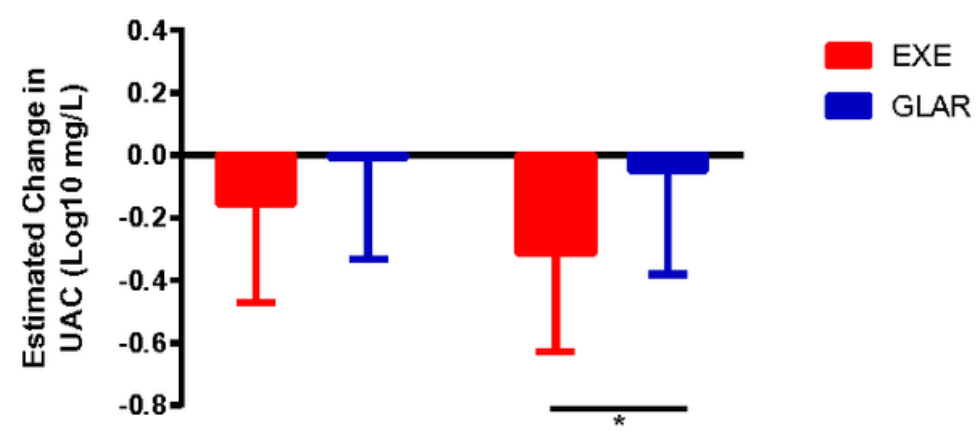

Week $12 \quad$ Week 24

D

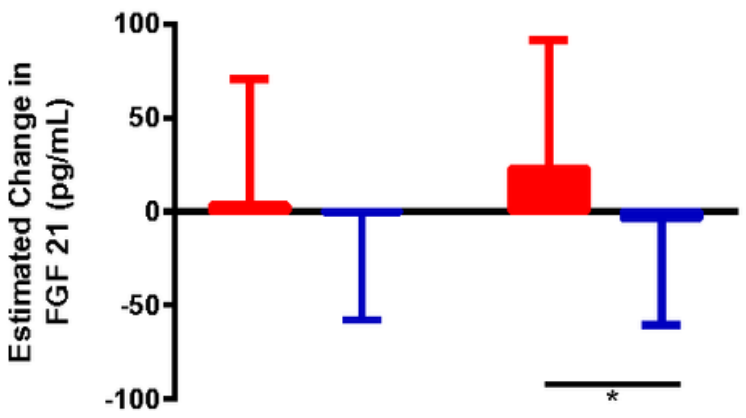

Week 12

Figure 2

Comparison of changes in UAC and FGF 21 within and between the groups. The linear mixed-effects model was used to compare within-group and intergroup changes of UAC (A-B) and FGF 21 (C-D) with gender as covariant. Data are presented as means $\pm S D$., ${ }^{*} P$ $<.001$. 
A

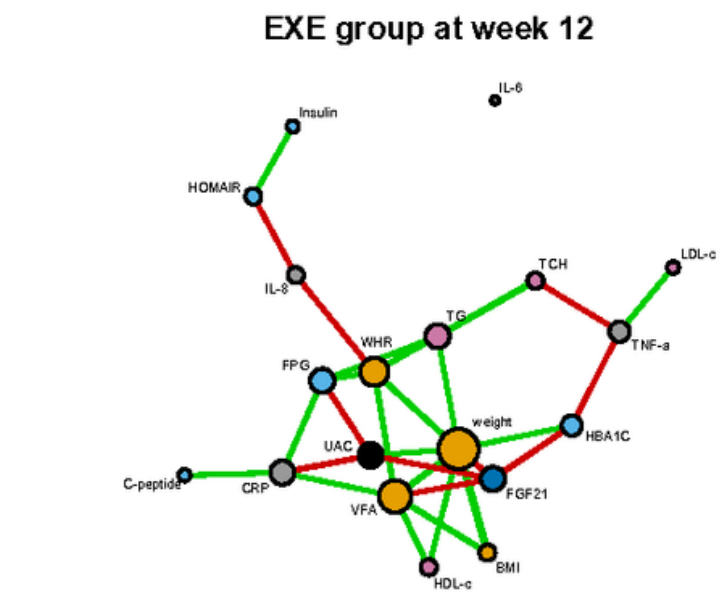

C

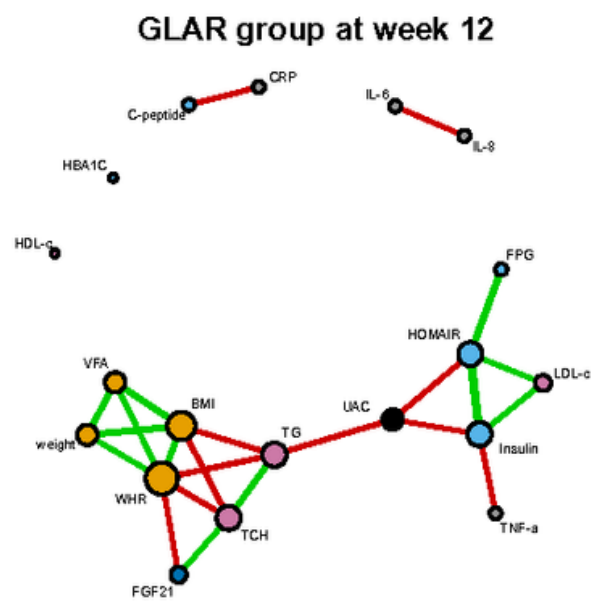

B

EXE group at week 24

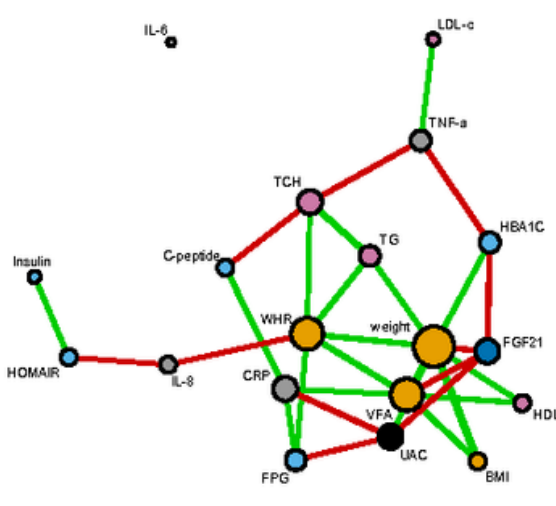

D

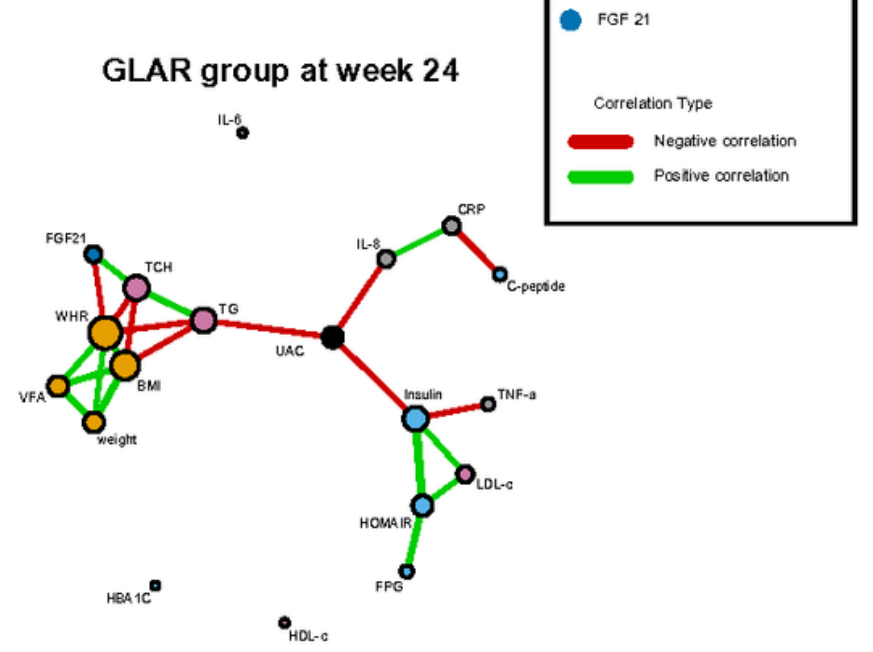

Figure 3

Correlation-based network analysis. Network visualization of correlations in the EXE group (A-B) and the GLAR group (C-D) at week 12 and week 24. Metabolic parameters are displayed as nodes and color-coded according to the type of parameters. UAC, anthropometric parameters, glucose and lipid metabolism parameters, inflammation parameters, and FGF 21 are displayed as black, yellow, blue, grey, pink, and dark blue nodes. The thickness of edges linking different variables represents significant positive (green) or negative (red) correlation coefficients. Abbreviations: BMI, body mass index; WHR, waist-hip ratio; VFA, visceral fat area; FBG, fasting blood glucose; HbA1C, glycosylated hemoglobin; TCH, total cholesterol; TG, triglycerides; LDL-c, low-density lipoprotein cholesterol, HDL-c, highdensity lipoprotein cholesterol; CRP, C-reactive protein; IL-6, interleukin-6; IL-8, interleukin-8; TNF-a, tumor necrosis factor-a.

\section{Supplementary Files}

This is a list of supplementary files associated with this preprint. Click to download.

- SupplementaryFigure1.pdf

- CONSORT2010Checklist.doc

- Protocol.doc 